\title{
Atom ionization by strong laser pulses - gauge invariant description
}

\author{
M. Ślęczka, A. Bechler, \\ Insitute of Physics, University of Szczecin, Wielkopolska 15, 70-451 Szczecin, Poland
}

Received October 17, 2013; accepted December 20, 2013; published December 31, 2013

\begin{abstract}
We present recent the results of our studies on matter interaction with strong laser pulses. It has been known that the results obtained with the use of Strong Field Approximation, which underlay the Keldysh-Faissal-Reiss theory (KFR), lead to a gauge dependent expression for probability amplitude. We propose a new approach to this problem and develop a new method to obtain an expression which is gauge independent. We present some examples of numerical calculations which show good agreement of our model with TDSE results.
\end{abstract}

Interaction of atomic and molecular systems with strong and short pulses of laser radiation has been intensively investigated for more than two decades. The process which attracts much attention, both from theoretical and experimental point of view, is an atomic and molecular photoeffect driven by strong pulsed radiation. A standard description of this phenomenon is based on Strong Field Approximation (SFA) known also as Keldysh-FaisalReiss theory [1]-[3]. As opposed to a standard perturbation method, SFA accounts for the interaction with an external field in a non-perturbative manner, and in the first approximation, neglects the influence of the atomic potential on the final continuum state of the electron.

SFA is a proper theoretical tool used for the description of laser-matter interaction in a strong field limit, ie. when $\omega<<\mathrm{U}_{\mathrm{p}}$ and $\omega<<\mathrm{I}_{\mathrm{p}}$ (where $\mathrm{U}_{\mathrm{p}}$ is the ponderomotive energy and $I_{p}$ is the binding energy). However, in general, its predictions depend on a chosen gauge of the scalar and vector potentials describing an external electromagnetic field. Considering the two most popular gauges, velocity(VG) and length-gauge (LG), theoretical predictions may differ by few orders of magnitude [4]-[6]. It seems that length gauge gives better agreement with experimental results and solution of the time dependent Schrödinger equation (TDSE). However, Reiss [5] showed that for detachment from negative fluorine ions, the velocity gauge is in better agreement with experimental measurements. It is certainly undesirable to be forced to choose an appropriate gauge depending, for example, on the pulse parameters. The main motivation of the present investigation is to develop a gauge-invariant description of the atomic interaction with short and strong laser pulses.

\footnotetext{
*E-mail: adamb@univ.szczecin.pl
}

We can write the Hamiltonian of the system interacting with strong and short laser pulses using a dipole approximation (atomic units are used throughout unless otherwise stated)

$$
\widehat{H}(t)=\widehat{H}_{0}+\widehat{V}_{a t}+\widehat{F}(t)
$$

where $\widehat{H}_{0}$ is the free Hamiltonin, $\widehat{V}_{a t}$ is the timeindependent atomic potential and $\hat{F}(t)$ describes the interaction of the electron with strong laser pulses. The Hamiltonian (1) can be partitioned in two ways

$$
\widehat{H}(t)=\widehat{H}_{a t}+\widehat{F}(t)=\widehat{H}_{F}(t)+\widehat{V}_{a t},
$$

where

$$
\begin{gathered}
\widehat{H}_{a t}=\widehat{H}_{0}+\widehat{V}_{a t}, \\
\widehat{H}_{F}=\widehat{H}_{0}+\widehat{F}(t),
\end{gathered}
$$

Using two types of the Hamiltonian (2) we can write two types of integral Dyson equations for the time evolution operator [7]

$$
\begin{gathered}
\widehat{U}\left(t, t^{\prime}\right)=\widehat{U}_{a t}\left(t, t^{\prime}\right)-i \int_{t^{\prime}}^{t} d t_{1} \widehat{U}\left(t, t_{1}\right) \widehat{F}\left(t_{1}\right) \widehat{U}_{a t}\left(t_{1}, t^{\prime}\right), \\
\widehat{U}\left(t, t^{\prime}\right)=\widehat{U}_{F}\left(t, t^{\prime}\right)-i \int_{t^{\prime}}^{t} d t_{1} \widehat{U}\left(t, t_{1}\right) \widehat{V}_{a t} \widehat{U}_{F}\left(t_{1}, t^{\prime}\right)
\end{gathered}
$$

where $\widehat{U}_{a t}$ and $\widehat{U}_{F}$ are the time evolution operators generated, respectively, by $\widehat{H}_{a t}$ and $\widehat{H}_{F}$ (3). We consider matter interaction with a laser pulse of finite time duration, starting at an initial time $t_{i}$ and terminating at a final time $t_{f}$. The time-dependent transition amplitude can be written as

$$
M=\left\langle\psi_{f}\left(t_{f}\right)\left|\widehat{U}\left(t_{f}, t_{i}\right)\right| \psi_{i}\left(t_{i}\right)\right\rangle,
$$

with $\left|\psi_{f}\right\rangle$ and $\left|\psi_{i}\right\rangle$ denoting the final continuum state of the ejected electron and initial bound state, respectively. With the use of the first Dyson equation (3a) we can rewrite (4) in the form 


$$
M_{f i}=-i \int_{t_{i}}^{t_{f}} d t_{1}\left\langle\psi_{f}\left(t_{f}\right)\left|\widehat{U}\left(t_{f}, t_{1}\right) \hat{F}\left(t_{1}\right)\right| \psi_{i}\left(t_{1}\right)\right\rangle .
$$

Standard form of SFA is obtained by approximating time evolution operator $\widehat{U}\left(t_{f}, t_{1}\right) \approx \widehat{U}_{F}\left(t_{f}, t_{i}\right)$ and the initial state by a plane wave. The ionization amplitude can be written as [7]

$$
M_{f i}=-i \int_{t_{i}}^{t_{f}} d t_{1}\left\langle\psi_{f}^{a}\left(t_{f}\right)\left|\widehat{U}_{F}\left(t_{f}, t_{1}\right) \hat{F}\left(t_{1}\right)\right| \psi_{i}\left(t_{1}\right)\right\rangle,
$$

in either gauge, where $\left\langle\psi_{f}^{a}\left(t_{1}\right)\right| \widehat{U}_{f}\left(t_{f}, t_{1}\right)$ is the Volkov state. The two most popular gauges are the velocity gauge, characterized by

$$
\hat{F}_{V}(t)=\widehat{\boldsymbol{p}} \cdot \boldsymbol{A}(t)+\frac{1}{2} \boldsymbol{A}^{2}(t),
$$

where $\boldsymbol{A}(t)$ is the vector potential of electromagnetic field, and the length gauge

$$
\widehat{F}_{L}(t)=\boldsymbol{r} \cdot \boldsymbol{E}(t),
$$

where $\boldsymbol{E}(t)$ denotes the electric field of the pulse, $\boldsymbol{E}(t)=-\partial \boldsymbol{A}(t) / \partial t$. Equation (6) can be written as [8]

$$
\begin{aligned}
& M_{0}= \\
& \begin{array}{l}
\left\langle\psi_{f}^{a}\left(t_{f}\right)\left|\widehat{U}_{F}\left(t_{f}, t_{i}\right)\right| \psi_{i}\left(t_{i}\right)\right\rangle-\left\langle\psi_{f}^{a}\left(t_{f}\right) \mid \psi_{i}\left(t_{i}\right)\right\rangle \\
-i \int_{t_{i}} d t_{1}\left\langle\psi_{f}^{a}\left(t_{f}\right)\left|\widehat{U}_{F}\left(t_{f}, t_{1}\right) \widehat{V}_{a t}\right| \psi_{i}\left(t_{1}\right)\right\rangle
\end{array}
\end{aligned}
$$

Expression (8) is gauge dependent since $\widehat{U}_{F}\left(t_{f}, t_{1}\right)$ depends on the choice of gauge for transient times.

A gauge invariant expression for the transition amplitude can be obtained by taking into account the next iteration of (3a)

$$
\begin{gathered}
M_{1}=(-i)^{2} \int_{t_{i}}^{t_{f}} d t_{1} \int_{t_{1}}^{t_{f}} d t_{2} \\
\left\langle\psi_{f}^{a}\left(t_{f}\right)\left|\widehat{U}_{F}\left(t_{f}, t_{2}\right) \widehat{V}_{a t} \widehat{U}_{F}\left(t_{2}, t_{1}\right) \hat{F}\left(t_{1}\right)\right| \psi_{i}\left(t_{i}\right)\right\rangle
\end{gathered}
$$

The right side of this equation can be transformed in an analogous way as (8) [8]

$$
\begin{gathered}
M_{1}=i \int_{t_{i}}^{t_{f}} d t_{2}\left\langle\psi_{f}^{a}\left(t_{f}\right)\left|\widehat{U}_{f}\left(t_{f}, t_{2}\right) \widehat{V}_{a t}\right| \psi_{i}\left(t_{2}\right)\right\rangle- \\
i \int_{t_{i}}^{t_{f}} d t_{2}\left\langle\psi_{f}^{a}\left(t_{f}\right)\left|\widehat{U}_{f}\left(t_{f}, t_{2}\right) \widehat{V}_{a t} \widehat{U}_{f}\left(t_{2}, t_{i}\right)\right| \psi_{i}\left(t_{i}\right)\right\rangle \\
+\cdots
\end{gathered}
$$

The first term of (10) is gauge dependent and it cancels with the gauge dependent contribution in (8) and the second term in (10) is independent of the choice of gauge. The term not shown explicitly in (10) is of the second order in atomic potential. Therefore, up to terms of the first order in atomic potential we can write a gauge independent expression for transition amplitude in the form

$$
M^{(0)}=\left\langle\psi_{f}^{a}\left(t_{f}\right)\left|\widehat{U}_{F}\left(t_{f}, t_{i}\right)\right| \psi_{i}\left(t_{i}\right)\right\rangle-\left\langle\psi_{f}^{a}\left(t_{f}\right) \mid \psi_{i}\left(t_{i}\right)\right\rangle,
$$

$M^{(1)}=-i \int_{t_{i}}^{t_{f}} d t_{1}\left\langle\psi_{f}^{a}\left(t_{f}\right)\left|\widehat{U}_{f}\left(t_{f}, t_{1}\right) \widehat{V}_{a t} \widehat{U}_{f}\left(t_{1}, t_{i}\right)\right| \psi_{i}\left(t_{i}\right)\right\rangle$.

Expressions (11) for the ionization amplitude were used for the analysis of energy spectra of photoelectrons emitted from the ground state of the hydrogen atom. The results were compared with TDSE results obtained with the use of the QPROP package [9]. We use a plane wave as an approximation for the final state of a photoelectron. This is justified for a short range potential and in the case of ionic potentials with a long-range Coulomb tail this approximation can be used for more energetic photoelectrons. The linearly polarized laser pulse is characterized by an electric field with a cosine squared envelope

$$
\boldsymbol{E}(t)=\left\{\begin{array}{cc}
\boldsymbol{e} E_{0}(t) \cos ^{2}\left(\frac{\pi t}{\tau}\right) \cos (\omega t+\varphi) & \text { for }-\frac{\tau}{2}<t<\frac{\tau}{2} \\
0 & \text { otherwise }
\end{array}\right.
$$

where $E_{0}$ is the electric field amplitude, $\tau$ is the pulse duration time and $\varphi$ is the carrier envelope phase (CEP). The laser pulse duration time $\tau$ is defined by $n T$, where $n$ is the number of optical cycles and $T$ is the carrier wave period determined by the carrier-wave frequency $\omega$. We present energy distribution of detached photoelectrons for a few different values of parameters, $E_{0}$ equal to 1 a.u. (it corresponds to the intensity equal to $3.5 \cdot 10^{20} \mathrm{~W} \cdot \mathrm{m}^{-2}$ ) and 3 a.u. (intensity equal to $3.15 \cdot 10^{21} \mathrm{~W} \cdot \mathrm{m}^{-2}$ ). We consider atom interaction with a laser pulse with the wave length $\lambda=80 \mathrm{~nm}(\omega=0.57$ a.u. $)$ with CEP equal to zero. Keldysh parameter, which determines which process, tunnell ionization or above threshold ionization, can occur, is smaller than one $\left(0.57\right.$ for $E_{0}=1$ a.u. and 0.19 for $E_{0}=3$ a.u.). We consider photoelectrons emitted in the direction of laser field polarization.

Previously, we performed the calculations for $E_{0}=1, n=10$ and $E_{0}=3, n=2$ [10]. The results of this calculation together with comparison with the TDSE predictions are shown here in Figs $1 \mathrm{a}$ and 1b, respectively. In general, we observe qualitative agreement between the present approach and the "exact" results 
based on a numerical solution of TDSE. The spectra show, in general, complicated structures which are due to interference effects. The present approach leads to good qualitative agreement with TDSE-results for the pulse parameters basically beyond the region of applicability of standard SFA, and also for larger energies of the emitted electron.
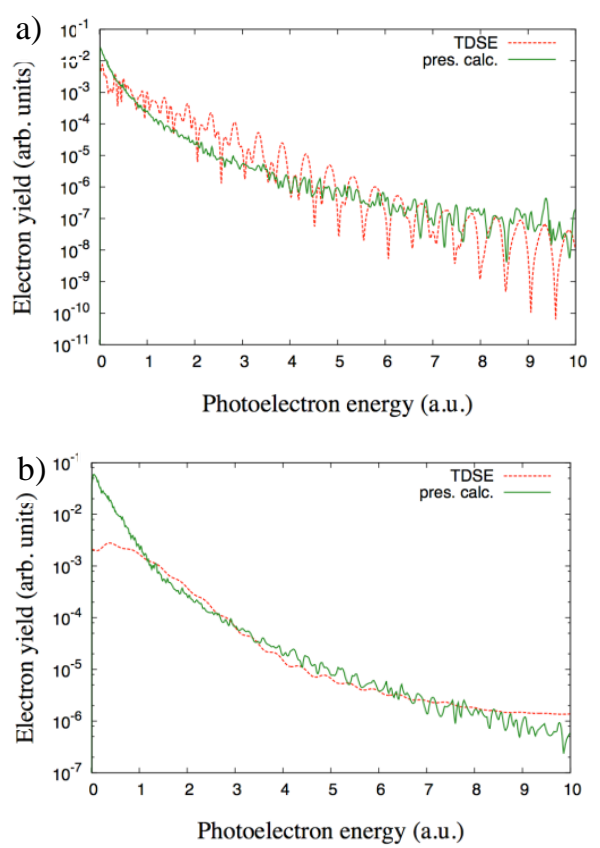

Fig. 1. Energy distribution of photoelectrons for $\omega=0.57$ a.u., a) $E_{0}=$ 1 a.u. and n $=10$, b) $E_{0}=3$ a.u. and $n=2$. Dashed red line corresponds to TDSE results, dashed green line corresponds to present calculations.

We also note that energy spectra show rather weak dependence on the pulse parameters, like electric field amplitude and number of optical wave - this is demonstrated in Fig. 2, where we show spectra for five different values of $E_{0}$ and $n$. It can be noticed that the spectra for different values of these parametres show small differences, except for some oscilations which occur in the region of higher energy of photoelectrons.

We have presented the results of our research on the laser-matter interaction in a strong field limit and derivation of a gauge-invariant description of this proces. The presented approach does not require any special partition of the total Hamiltonian into a "free" and an "interacting" part, as in [11-12]. Our method is based on consequent grouping of all terms in the same order with respect to atomic potential, which leads to a gauge independent expression order-by-order. We have limited numerical calculations to the first order term and compared it with TDSE results. Our calculations show good qualitative agreement with TDSE results, especialy in the high photoelectron energy region.
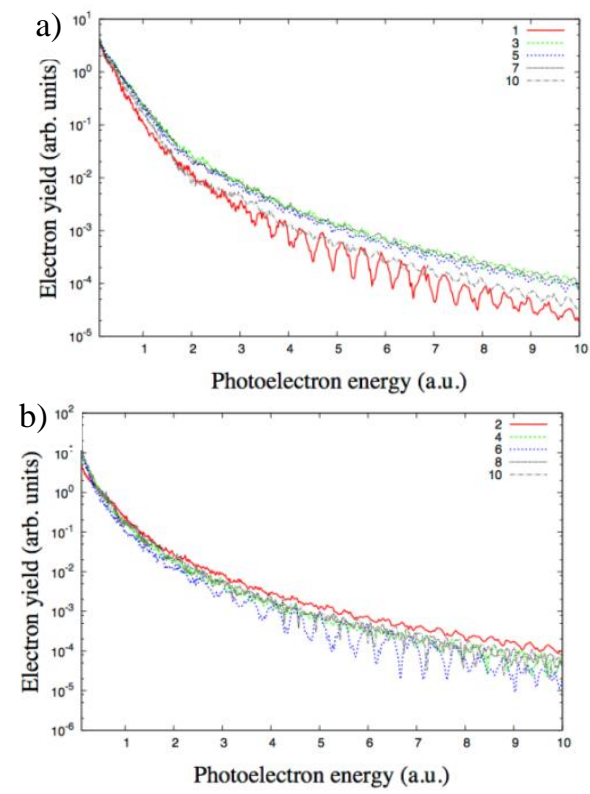

Fig. 2. Energy distribution of photoelectrons for $\omega=0.57$ a.u.,

a) different value of $E_{0}$ and $n=2$, b) $E_{0}=3$ a.u. and different value of $n$.

The present approach reproduces better the TDSE results for the values of parameters beyond the region of validity of standard SFA (for SFA $\omega \ll I_{p}, \omega \ll U_{p}$, where $I_{p}$ is the binding potential and $U_{p}$ is the ponderomotive energy). We have also observed that our model shows better agreement with TDSE results for a stronger field and higher energies.

This research was supported in part by PL-Grid Infrastructure.

\section{References}

[1] L.V. Keldysh, Zh. Eksp. Theor. Fiz. 47, 1945(1964). L.V. Keldysh, Sov. Phys. JETP 20137 (1965).

[2] F.H.M. Faisal, J. Phys. B; At. Mol. Phys. 6 L89 (1973), http://iopscience.iop.org/0022-3700/6/4/011.

[3] H.R. Reiss, Phys. Rev. A 22, 1786 (1980), http://link.aps.org/doi/10.1103/PhysRevA.22.1786.

[4] D. Bauer, D.B. Milošević, W. Becker, Phys. Rev. A 72023415 (2005), http://link.aps.org/doi/10.1103/PhysRevA.72.023415.

[5] H.R. Reiss, Phys. Rev. A 76033404 (2007), http://link.aps.org/doi/10.1103/PhysRevA.76.033404.

[6] J. Bauer, Phys. Rev. A 84025403 (2011), http://link.aps.org/doi/10.1103/PhysRevA.84.025403.

[7] W. Becker, F. Grasbon, R. Kopold, D.B. Milošević, G.G. Paulus, D. Bauer, Adv. At. Mol. Opt. Phys. 48, 35 (2002).

[8] A. Bechler, M. Ślęczka, Phys. Letters A 375, 1579 (2011), http://dx.doi.org/10.1016/j.physleta.2011.02.054.

[9] D. Bauer, P. Koval, Comp. Phys. Comm. 174(5) 396 (2006), http://dx.doi.org/10.1016/j.cpc.2005.11.001.

[10] A. Bechler, M. Slęczka, Phys. Rev. A 87, 025402 (2013), http://link.aps.org/doi/10.1103/PhysRevA.87.025402.

[11] F.H.M. Faisal, J. Phys. B: At. Mol. Opt. Phys. 40, F145 (2007).

[12] Y.V. Vanne, A. Saenz, Phys. Rev. A 79, 023421 (2009), http://link.aps.org/doi/10.1103/PhysRevA.79.023421. 